\title{
Etiología de la neumonía en pacientes chilenos infectados por el virus de la inmunodeficiencia humana
}

\author{
Carlos Pérez C., Patricia García C., Mario Calvo A., Jaime Labarca L., Marisol Bustos M., \\ Teresa Beroíza W., Pablo Gaete G., Rodrigo Moreno B., Guillermo Acuña L. y Pablo Vial C. .
}

\section{Etiology of pneumonia in Chilean HIV-infected adult patients}

Objectives: To establish the etiology of pneumonia and to compare the yield of diagnostic techniques for diagnosis of Pneumocystis jiroveci and Mycobacterium tuberculosis infections in HIV-1-infected patients. Patients and Methods: Subjects underwent sputum induction and bronchoalveolar lavage (BAL). Gram, Ziehl-Neelsen, silver stain (SS) and immunofluorescense staining (IF) for $P$. jiroveci, fluorescent stain for mycobacteria, PCR for $P$. jiroveci and $M$. tuberculosis, aerobic, fungal and mycobacterial cultures, respiratory viruses and CMV cultures were performed on the sputum and BAL. IgM for Mycoplasma pneumoniae and Chlamydophyla pneumoniae, and Legionella pneumophila urinary antigen were also obtained. Results: Sixty patients were included. An etiologic diagnosis was made in 97\%. Pneumocystis jiroveci was the most frequent etiology (58\%) followed by Streptococcus pneumoniae (12\%), and Mycobacterium avium complex (12\%). Mycobacterium tuberculosis was found in 5\%. Conclusions: The comparison of diagnostic methods for $P$. jiroveci showed a higher sensitivity of IF and SS in BAL than in sputum, however PCR was equally sensitive in both samples. With this approach a precise etiologic diagnosis was reached in the great majority of patients. The most common etiology was P. jiroveci. IF in BAL remains the gold standard for diagnosis of $P$. jiroveci pneumonia.

Key words: Pneumonia, Acquired Immunodeficiency Syndrome, HIV, AIDS-Related Opportunistic Infections, Etiology.

Palabras clave: Neumonía, síndrome de inmunodeficiencia adquirida, VIH, infecciones oportunistas, etiología.

\section{Introducción}

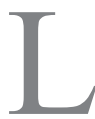

a neumonía es una causa importante de morbilidad y mortalidad entre los pacientes infectados por

VIH. En un estudio de 240 personas infectadas por VIH en Chile, la neumonía fue la enfermedad definitoria de SIDA en $50 \%$ de los pacientes ${ }^{1}$. El sistema respiratorio es un sitio donde frecuentemente se producen infecciones fúngicas (Pneumocystis jiroveci, anteriormente denominado Pneumocystis carinii), bacterianas y por micobacterias. La tasa de neumonía por $P$. jiroveci en los pacientes con recuentos de linfocitos CD4 $\leq$ a 200 céls $/ \mathrm{mm}^{3}$, sin uso de profilaxis, es de 8,08 casos por 100 personas-año ${ }^{2}$. La neumonía bacteriana se presenta con una incidencia entre 2,3 y 10,8 episodios por 100 personas-año en los pacientes infectados por VIH (comparado con 0,9 por 100 personasaño en individuos sanos) $)^{3,4}$. La broncoscopia con lavado bronco-alveolar (LBA) se considera el método estándar para el diagnóstico etiológico de la neumonía en pacientes infectados por VIH. No obstante, puesto que es un método invasor, el esputo inducido aparece como una estrategia diagnóstica sensible y costo-efectiva. Las nuevas técnicas de diagnóstico molecular podrían disminuir la necesidad de procedimientos invasores para el diagnóstico etiológico de la neumonía en estos pacientes.

El objetivo primario de este estudio fue establecer el diagnóstico etiológico de la neumonía en pacientes infectados por VIH utilizando métodos tradicionales y moleculares para la identificación de los microorganismos. El objetivo secundario fue comparar el rendimiento de diferentes técnicas diagnósticas en muestras de esputo inducido o espontáneo con las obtenidas por LBA para el diagnóstico de infecciones por $P$. jiroveci y Mycobacterium tuberculosis.

\section{Pacientes y Métodos}

\section{Población estudiada}

Entre octubre de 1999 y mayo de 2002, fueron invitados a participar pacientes infectados o con sospecha de infección por VIH que ingresaron por neumonía en forma consecutiva al Hospital Clínico de la Universidad Católica y al Hospital de Urgencia Alejandro Del Río en Santiago de Chile. Se definió neumonía como una infección respiratoria aguda caracterizada por fiebre,
Pontificia Universidad Católica de Chile Departamento de Medicina Interna (CPC,MCA, JLL, MBM, PGG, GAL). Departamento de Laboratorio Clínico (PGC).

Departamento de Enfermedades Respiratorias (TBW, RMB) Departamento de Pediatría (PVC). Programa de Enfermedades Infecciosas (CPC, PGC, JLL, GAL).

Afiliaciones actuales:

MCA: Universidad Austral de Chile, Valdivia, Chile.

MBM: Hospital DIPRECA,

Santiago, Chile.

PGG: Hospital Clínico Universidad

de Chile, Santiago

GAL: Clínica Las Condes,

Santiago, Chile.

PVC: Universidad del Desarrollo;

Santiago, Chile.

Proyecto financiado por

FONDECYT \# 1990119

Recibido: 23 de septiembre de 2010

Aceptado: 5 de junio de 2011

Correspondencia a:

Carlos Pérez Cortés

cape@med.puc.cl 
tos y/o expectoración, asociada a un infiltrado nuevo y persistente en la radiografía de tórax ${ }^{5}$. También se incluyeron pacientes con síntomas respiratorios agudos e insuficiencia respiratoria con radiografía inicial normal, siempre que posteriormente aparecieran infiltrados pulmonares radiográficos. El protocolo fue aprobado por los Comités de Ética de ambas instituciones y se obtuvo consentimiento informado de los pacientes antes del inicio de los procedimientos diagnósticos. Se excluyeron los pacientes con indicación de ventilación mecánica $(5 \%$ de los pacientes).

Al ingreso se consignó las características demográficas, sintomatología y signología clínica de los pacientes. Se obtuvieron recuentos hematológicos, niveles de deshidrogenasa láctica, gasometría arterial u oximetría de pulso y radiografía de tórax. Los recuentos de linfocitos CD4 y carga viral de VIH-1 fueron obtenidos de los registros clínicos de los enfermos o efectuados al ingreso.

\section{Protocolo de estudio}

En todos los pacientes se obtuvo una muestra de esputo espontáneo o inducido seguido por LBA.

Esputo inducido: Se hizo aseo de la cavidad bucal y se efectuó nebulización ultrasónica (Omron ${ }^{\circledR}$ NEU12) utilizando solución salina estéril al 3\% durante 15 minutos, con una frecuencia ultrasónica de 0,2 a $0,8 \mathrm{ml}$ por minuto. Se efectuaron ejercicios respiratorios, vibropresiones y ocasionalmente técnicas de drenaje bronquial en distintos decúbitos para la obtención de expectoración. Las muestras de esputo fueron evaluadas con tinción de Gram y estudio citológico para establecer su calidad, de acuerdo a los criterios de Murray ${ }^{6}$.

Lavado bronco-alveolar: Bajo sedación con anestesia local, se introdujo el fibro-broncoscopio a través de una fosa nasal o la boca hasta enclavar el instrumento en un bronquio segmentario. Se efectuó LBA con dos alícuotas de $60 \mathrm{ml}$ de $\mathrm{NaCl}$ al 9\%o estéril, aspirando el contenido a través de la misma jeringa. Las muestras se enviaron de inmediato al laboratorio para su procesamiento.

Las muestras de esputo y LBA fueron estudiadas en paralelo mediante tinciones de Gram, Ziehl-Neelsen y Gomori metenamina de plata (TGMP), IF directa para P. jiroveci (MeriFluor Pneumocystis, Meridian), tinción de auramina-rodamina para micobacterias (TB-color; Merck, Darmstadt, Germany), cultivos bacterianos aeróbicos convencionales (agar sangre de cordero al 5\%, agar chocolate y Mac Conkey), cultivos fúngicos (agar dextrosa Sabouraud) y cultivo de micobacterias (MB BacT sistema automatizado BioMerieux, Mo). Se efectuó reacción de polimerasa en cadena para $P$. jiroveci y $M$. tuberculosis en ambas muestras, según lo descrito en la literatura científica ${ }^{7,8}$. Se realizó cultivos de virus respiratorios (incluyendo virus respiratorio sincicial, influenza y parainfluenza, inoculando la muestra en células MDCK y Hep2 y tiñéndolas con anticuerpos monoclonales) y cultivos de citomegalovirus (CMV) (sólo en LBA, inoculados en fibroblastos). Se efectuó hemocultivos (BacT/Alert automated system ${ }^{\circledR}$, BioMerieux, Mo), IgM para Mycoplasma pneumoniae (immunofluorescencia indirecta, Wampole Laboratories) considerándose positiva una dilución mayor de 1:10, IgM para Chlamydophyla pneumoniae (microimmunofluorescencia, Vircell, Spain) y antígeno urinario de Legionella pneumophila (ELISA, Binax). A la muestra de esputo se le realizó detección de antígeno de Streptococcus pneumoniae ${ }^{9}$.

Las radiografías de tórax fueron informadas por radiólogo y discutidas con el investigador principal. Se reportaron los siguientes patrones radiológicos: normal, consolidación, infiltrado intersticial, mixto o nodular.

Los observadores que compararon el rendimiento del esputo y del LBA no estaban "ciegos" al tipo de muestra que se comparaba.

\section{Análisis estadístico}

La asociación entre variables fue estudiada mediante ANOVA o $\chi^{2}$ según el tipo de variable. El análisis fue conducido utilizando el software Epi-Info 6.04.

\section{Definiciones diagnósticas}

- Diagnóstico definitivo de neumonía por P. jiroveci: Una IF o TGMP en esputo y/o LBA positivo para $P$. jiroveci.

- Diagnóstico probable de neumonía por P. jiroveci: Una RPC positiva para $P$. jiroveci en esputo y/o LBA con cuadro clínico compatible con neumonía por $P$. jiroveci (LDH elevada y patrón radiológico mixto).

- Neumonía neumocóccica: Hemocultivos positivos y/o cultivo obtenido por LBA con desarrollo de S. pneumoniae. También pacientes previamente expuestos a antimicrobianos que tuvieran consolidación pulmonar y un examen de antígeno de $S$. pneumoniae positivo en esputo y/o LBA.

- Neumonía por M. pneumoniae y C. pneumoniae: Una serología de IgM positiva, en ausencia de otro diagnóstico etiológico.

\section{Resultados}

En el período definido se reclutaron 61 pacientes. Un caso no fue incluido en el análisis final ya que no se pudo realizar la fibro-broncoscopia por intolerancia del paciente al procedimiento. Se estudiaron 60 pacientes (57 hombres) con una edad media de 39,4 años (20-83 años). En tres de los 60 pacientes se concluyó que no tenían neumonía. En estos casos, los diagnósticos fueron miocarditis por Toxoplasma gondii con edema pulmonar agudo, sarcoma de Kaposi pulmonar e infección viral del tracto respiratorio superior, respectivamente. 
El análisis final incluyó 57 pacientes. El factor de riesgo para la adquisición del VIH fue la vía sexual en $100 \%$ de los casos (homosexual masculina en $78,3 \%$ ). La neumonía fue la primera manifestación de infección por VIH en 16 de los 57 pacientes (28\%). Sólo nueve (16\%) de los 41 pacientes con infección por VIH conocida estaban recibiendo terapia anti-retroviral (TARV) de alto grado de actividad, al momento de ingresar al estudio. Treinta $\mathrm{y}$ dos pacientes habían tenido infecciones oportunistas previas $(56 \%)$, destacando candidiasis (n: 23$)$ y herpes zoster (n: 4). Se obtuvo muestra de esputo en 56 de los 57 pacientes. La mayoría de los pacientes cursaba una etapa avanzada de la enfermedad, con un recuento de linfocitos

\begin{tabular}{|c|c|}
\hline & n (\%) de pacientes \\
\hline Recuento de linfocitos CD4 (céls/mm³) & (n: 54) \\
\hline$<50$ & $24(44,4)$ \\
\hline $51-100$ & $9(16,7)$ \\
\hline $100-200$ & $12(22,2)$ \\
\hline$>200$ & $9(16,7)$ \\
\hline Promedio \pm DS (rango) & $115,9 \pm 126,3(4-505)$ \\
\hline Carga viral & (n: 56) \\
\hline$>100.000$ copias ARN/mL & $38(68)$ \\
\hline$<50$ copias ARN/mL & $6(11)$ \\
\hline
\end{tabular}

Signos y síntomas:

$\begin{array}{ll}\text { Fiebre } & 51(89,5) \\ \text { Calofríos } & 25(43,9) \\ \text { Sudoración } & 28(49,1) \\ \text { Tos } & 51(89,5) \\ \text { Expectoración } & 33(66,1) \\ \text { Disnea } & 42(73,7) \\ \text { Dolor torácico } & 12(21,1) \\ \text { Examen pulmonar anormal } & 34(59,6)\end{array}$

\begin{tabular}{lr|} 
Laboratorio: & \\
Hipoxemia & $21(36,8)$ \\
Leucocitosis $>9.000$ céls $/ \mathrm{mm}^{3}$ & $12(21,1)$ \\
$\mathrm{LDH}>225 \mathrm{UI} / \mathrm{mL}$ & $43(75,4)$ \\
\hline Patrón radiológico & $2(3,5)$ \\
Normal & $22(38,6)$ \\
Consolidación & $38(66,7)$ \\
Infiltrado intersticio-alveolar & $7(12,3)$ \\
Nódulos & $4(7)$ \\
Efusión pleural &
\end{tabular}

CD4 bajo 200 céls $/ \mathrm{mm}^{3}$ en $83 \%$ de los casos. De los nueve pacientes en TARV, seis tenían recuento de linfocitos CD4 bajo 200 céls $/ \mathrm{mm}^{3}$ y siete tenían carga viral indetectable. Las manifestaciones clínicas más frecuentes de neumonía fueron fiebre $(89,5 \%)$, tos $(89,5 \%)$, disnea $(73,7 \%), \mathrm{LDH}$ elevada $(75,4 \%)$ y un patrón radiográfico con infiltrados mixtos en $66,7 \%$ (Tabla 1 ). No hubo fallecimientos.

\section{Etiología de la neumonía}

Se obtuvo un diagnóstico etiológico en $96,5 \%$ de los pacientes. Las causas más frecuentes de neumonía fueron P. jiroveci, seguido por S. pneumoniae, Mycobacterium avium complex (MAC) (co-existiendo con otros microorganismos en tres de siete casos) y Staphylococcus aureus (co-existiendo con otros agentes en dos de cuatro casos) (Tabla 2). El 50\% de los pacientes tenía cultivos positivos para CMV sin un cuadro clínico consistente con este agente. Ningún paciente requirió de tratamiento específico para este virus.

Pneumocystis jiroveci fue la etiología más común de neumonía. Excepto por la alta frecuencia de producción de esputo $(66,6 \%)$ y las alteraciones encontradas al examen pulmonar $(63,3 \%)$, el cuadro clínico fue similar al patrón clásico descrito para este agente (Tabla 3). Comparado con otras etiologías, los pacientes con neumonía por $P$. jiroveci presentaban una mayor frecuencia de patrón mixto en la radiografía de tórax $(p<0,005)$ y una mayor

\begin{tabular}{|c|c|}
\hline & n (\%) de pacientes \\
\hline Pneumocystis jiroveci (definitivo) & $30(52,6)$ \\
\hline Pneumocystis jiroveci (probable) & $3(5,2)$ \\
\hline Streptoccoccus pneumoniae & $7(12,3)$ \\
\hline Mycobacterium avium - complex & $7(12,3)$ \\
\hline Staphylococcus aureus & 4 (7) (2 MRSA) \\
\hline Mycobacterium tuberculosis & $3(5,3)$ \\
\hline Influenza A & $2(3,5)$ \\
\hline Cryptococcus neoformans & $1(1,8)$ \\
\hline Acinetobacter baumannii & $1(1,8)$ \\
\hline Streptococcus agalactiae & $1(1,8)$ \\
\hline Haemophilus influenzae & $1(1,8)$ \\
\hline Mycoplasma pneumoniae & $1(1,8)$ \\
\hline Chlamydophyla pneumoniae & $1(1,8)$ \\
\hline Estudio microbiológico negativo & $2(3,5)$ \\
\hline \multicolumn{2}{|c|}{$\begin{array}{l}\text { *Un agente causal en } 50 \text { pacientes }(87,7 \%) \text {. Más de un agente } \\
\text { causal en } 5 \text { pacientes }(8,8 \%) \text {. Cultivo positivo para CMV en LBA en } \\
28 \text { pacientes }(50 \%)\end{array}$} \\
\hline
\end{tabular}




\begin{tabular}{|c|c|}
\hline & n (\%) de pacientes \\
\hline \multicolumn{2}{|c|}{ Recuento de linfocitos CD4 (céls/mm³): } \\
\hline$<50$ & $16(53,3)$ \\
\hline$<200$ & $29(96,7)$ \\
\hline$>200$ & $1(3,3)$ \\
\hline \multicolumn{2}{|l|}{ Carga viral (copias ARN/mL) } \\
\hline$<50$ & $1(3,3)$ \\
\hline$>100.000$ & $27(90)$ \\
\hline Infecciones oportunistas previas & $15(50)$ \\
\hline HAART & $1(3,3)$ * \\
\hline Profilaxis neumonía $P$. jiroveci & 0 \\
\hline \multicolumn{2}{|l|}{ Cuadro clínico: } \\
\hline Fiebre & $25(83,3)$ \\
\hline Tos & $28(93,3)$ \\
\hline Expectoración & $20(66,6)$ \\
\hline Disnea & $27(90)$ \\
\hline Examen pulmonar anormal & $19(63,3)$ \\
\hline $\mathrm{LDH}>225 \mathrm{UI} / \mathrm{mL}$ & $26(86,6)$ \\
\hline Hipoxemia & $12(40)$ \\
\hline \multicolumn{2}{|l|}{ Patrón radiológico: } \\
\hline Infiltrado intersticio-alveolar & $28(93,3)$ \\
\hline Normal & $1(3,3)$ \\
\hline Consolidación & $1(3,3)$ \\
\hline
\end{tabular}

Tabla 4. Sensibilidad, especificidad, valores predictores positivo y negativo de la tinción fluorescente, tinción de Gomori metenamina plata y reacción de polimerasa en cadena para Pneumocystis firoveci en muestras de expectoración y lavado bronco-alveolar en 30 pacientes con infección por VIH y neumonía por $P$. jiroveci

\begin{tabular}{|lcccccc|} 
& & & Expectoración & \multicolumn{3}{c}{ Lavado bronco-alveolar } \\
& IF & TGMP & RPC & IF & TGMP & RPC \\
\hline Sensibilidad & 70 & 16,7 & 96,7 & 96,7 & 56,7 & 96,7 \\
Especificidad & 92 & 100 & 69,2 & 100 & 100 & 63 \\
\hline VPP & 92,3 & 100 & 78,4 & 100 & 100 & 74,4 \\
VPN & 72,7 & 51 & 94,7 & 96,4 & 67,5 & 94,4 \\
\hline
\end{tabular}

IF: inmunofluorescencia. TGMP: tinción de Gomori metenamina plata. RPC: reacción de polimerasa en cadena. VVP: valor predictor positivo. VVN: valor predictor negativo. frecuencia de niveles de deshidrogenada láctica sobre $225 \mathrm{UI} / \mathrm{ml}(\mathrm{p} \mathrm{0,02)}$.

El esputo fue comparado con el LBA respecto al rendimiento de los diferentes métodos diagnósticos para $P$. jiroveci (Tabla 4). La IF presentó una menor sensibilidad en esputo (70\%) que en el LBA $(96,7 \%)$. La TGMP tuvo un menor rendimiento en esputo que en LBA (16,7 y $56,7 \%$ respectivamente). La RPC para $P$. jiroveci mostró una alta sensibilidad en ambas muestras; sin embargo, la especificidad fue baja puesto que hubo pacientes con otros diagnósticos que presentaron un test positivo en esputo (8 casos) o LBA (10 pacientes), pero que mejoraron sin tratamiento específico para $P$. jiroveci y, por lo tanto, se consideraron colonizados por este agente.

En nuestro estudio se diagnosticó tuberculosis a tres pacientes; todos tuvieron tinciones de Ziehl-Neelsen y auramina-rodamina, RPC y cultivo de micobacterias, positivos en esputo. En LBA las tinciones de ZiehlNeelsen y auramina-rodamina fueron positivas en dos de tres pacientes. La RPC para M. tuberculosis y el cultivo de micobacterias fueron positivos en los tres casos. En todos los pacientes con infección por MAC, la tinción de micobacterias y la RPC para M. tuberculosis en esputo y LBA fueron negativas.

\section{Discusión}

El uso de profilaxis para infecciones oportunistas, y más recientemente, la disponibilidad de TARV, han cambiado la historia natural de la infección por VIH ${ }^{10,11}$. Sin embargo, las complicaciones siguen constituyendo una causa significativa de morbilidad y mortalidad en este grupo de pacientes ${ }^{12}$. Nosotros estudiamos 57 pacientes infectados por VIH-1, hospitalizados por neumonía adquirida en la comunidad. La neumonía fue la primera manifestación de infección por VIH en un tercio de los casos, la gran mayoría de los pacientes tenía un recuento de CD4 $\leq 200$ céls $/ \mathrm{mm}^{3}$ y sólo $16 \%$ de los pacientes estaban recibiendo TARV. Esta situación ha cambiado en Chile y actualmente la mayoría de los pacientes con enfermedades oportunistas o recuentos de $\mathrm{CD} 4<\mathrm{a} 200$ céls/ $\mathrm{mm}^{3}$ se encuentra recibiendo TARV ${ }^{13}$. No obstante, aún en la actualidad, un número significativo de pacientes debuta en etapa $\mathrm{C}$ debido a un diagnóstico tardío de la infección (Cortés y cols. XXIV Congreso Chileno de Infectología, Chile). La baja aplicación de la profilaxis para neumonía por $P$. jiroveci se explica porque no habiendo TARV, la mayoría de los pacientes no se encontraba bajo control médico al momento de presentar la neumonía.

Con nuestra aproximación diagnóstica logramos un diagnóstico específico en $96,5 \%$ de los pacientes. La etiología más frecuente fue la infección por $P$. jiroveci seguida por S. pneumoniae. Estos resultados son muy 
similares a lo descrito con anterioridad a los inicios de la TARV ${ }^{4}$. Mycobacterium avium complex compartió el segundo lugar como agente etiológico por frecuencia (co-existiendo con otros agentes en 3/7 de los casos); no obstante, es infrecuente que este agente constituya una causa primaria de neumonía y habitualmente su aislamiento representa colonización o enfermedad diseminada ${ }^{14,15}$.

Citomegalovirus fue aislado en los cultivos de LBA en $50 \%$ de los casos; sin embargo, en todos los pacientes se identificó algún diagnóstico alternativo y mejoraron sin requerir tratamiento específico, tal como ha descrito por varios autores ${ }^{16,17}$. La tuberculosis pulmonar fue diagnosticada en sólo tres pacientes, probablemente debido a que la tuberculosis en Chile ha bajado durante la última década llegando a 15,7 casos por cien mil habitantes en el año $2005^{18}$.

Hubo dos casos de neumonía atípica por C. pneumoniae y $M$. pneumoniae, respectivamente, y ningún caso de neumonía por Legionella spp. Si bien sólo utilizamos IgM, para el diagnóstico de C. pneumoniae y $M$. pneumoniae y detección de antígeno urinario para Legionella spp, nuestros resultados son comparables con lo descrito por otros autores quienes reportan una menor incidencia de estos agentes en los pacientes con infección por VIH, comparado con pacientes inmunocompetentes ${ }^{19,20}$.

Un estudio chileno retrospectivo reciente incluyó a 171 pacientes infectados con VIH que presentaron 236 episodios de enfermedad pulmonar aguda entre 1999 y 2003. Al igual que en nuestro estudio, sólo 13,5\% de los pacientes estaban recibiendo TARV. Las causas más frecuentes de neumonía fueron $P$. jiroveci, seguido por bacterias incluyendo micobacterias. El grupo también incluyó a 14 neumonías nosocomiales por bacterias resistentes y no se estudió en forma sistemática la presencia de virus respiratorios o agentes atípicos. Tampoco se efectuó en forma rutinaria LBA o estudios moleculares para el diagnóstico microbiológico ${ }^{21}$.

En nuestra serie, $P$. jiroveci es el agente etiológico más frecuente. La presentación clínico-radiográfica es similar a la descripción clásica del cuadro, excepto porque $2 / 3$ de los pacientes tuvieron expectoración y examen pulmonar anormal. Con respecto al rendimiento de las técnicas utilizadas para el diagnóstico de $P$. jiroveci, encontramos que la IF fue más sensible que la TGMP en las muestras de esputo (70 versus $17 \%$, respectivamente), resultados similares a lo descrito en el meta-análisis de Cruciani y $\operatorname{cols}^{22}$. Los valores predictores positivos y negativos de la IF y TGMP en muestras de esputo varían de acuerdo a la prevalencia de infección por $P$. jiroveci, pero nuestros resultados son comparables a lo estimado en este metaanálisis. La sensibilidad de la IF (97\%) y TGMP (57\%) en LBA fueron mayores que las encontradas en esputo inducido. La RPC para $P$. jiroveci tuvo una sensibilidad cercana a $100 \%$, tanto en esputo como LBA. La especi- ficidad cayó debido a que algunos pacientes tuvieron un diagnóstico etiológico diferente $\mathrm{y}$, por lo tanto, estaban colonizados por $P$. jiroveci, tal como ha sido extensamente descrito en la literatura médica ${ }^{23-26}$. Algunos de estos pacientes podrían desarrollar infección por $P$. jiroveci a futuro, según lo reportado por Elvin y cols ${ }^{27}$, quienes describieron que seis de ocho pacientes asintomáticos con una RPC positiva para $P$. jiroveci, desarrollaron neumonía por este agente entre los 164 y 352 días posteriores al resultado positivo del examen. La RPC también puede realizarse en muestras obtenidas de enjuagues orales para el diagnóstico de neumonía por $P$. jiroveci ${ }^{28}$.

El pequeño número de casos de infección por $M$. tuberculosis no nos permite comparar el rendimiento de las diferentes técnicas diagnósticas; sin embargo, debemos señalar que la sensibilidad y especificidad de las tinciones de Ziehl-Neelsen y auramina-rodamina, así como la RPC, fueron excelentes y no se observaron falsos positivos en los pacientes con infecciones por MAC.

En conclusión, en esta cohorte de pacientes chilenos con infección por VIH-1 hospitalizados por neumonía, la etiología más frecuente fue $P$. jiroveci, seguida por $S$. pneumoniae y MAC. Nuestros resultados muestran que la IF en LBA sigue siendo el estándar de oro para el diagnóstico de $P$. jiroveci. La RPC para $P$. jiroveci tiene una alta sensibilidad en esputo y LBA; sin embargo, la especificidad es menor debido a que algunos pacientes están colonizados por este agente. La infección por CMV asintomática es frecuente. Nuestros resultados son aplicables en nuestro país, aún en la era de la TARV, dada la gran cantidad de pacientes que debuta en etapas avanzadas de la enfermedad.

Agradecimientos. A todo el equipo de Kinesiología del Hospital Clínico de la Universidad Católica y del Hospital de Urgencia Alejandro Del Río en Santiago de Chile, por la implementación de la técnica de esputo inducido.

\section{Resumen}

Objetivos: Establecer la etiología de la neumonía y comparar el rendimiento de diferentes técnicas para el diagnóstico de las infecciones por Pneumocystis jiroveci y Mycobacterium tuberculosis en pacientes con infección por virus de inmunodeficiencia humana (VIH). Material y Métodos: De cada paciente se obtuvo esputo inducido y se efectuó LBA. A las muestras obtenidas se les realizó tinciones de Gram, Ziehl-Neelsen, plata e inmunofluorescencia (IF) para $P$. jiroveci y $M$. tuberculosis; reacción de polimerasa en cadena (RPC) para ambos microorganismos; cultivos aeróbicos, fúngicos, para micobacterias, virus respiratorios y citomegalovirus. También se realizó determinación de IgM de Mycoplasma pneumoniae y Chlamydophyla pneumoniae y antígeno urinario de 
Legionella pneumophila. Resultados: Se incluyeron 60 pacientes, lográndose diagnóstico etiológico en $97 \%$ de los casos. Pneumocystis jiroveci fue la etiología más frecuente (58\%), seguida por Streptococcus pneumoniae (12\%) y Mycobacterium avium complex (MAC) (12\%). Mycobacterium tuberculosis fue encontrado en 5\%. Conclusiones: La comparación de los métodos diagnósticos para $P$. jiroveci mostró una mayor sensibilidad de la IF y tinción de plata en LBA que en esputo; sin embargo, la RPC fue igualmente sensible en ambos tipos de muestras. Con esta estrategia se logró establecer etiología en la gran mayoría de los pacientes. La etiología más común fue $P$. jiroveci. IF en LBA sigue siendo el estándar para el diagnóstico de la neumonía por $P$. jiroveci.

\section{Referencias}

1.- Wolff M, Northland R, Segovia J, Beltrán C, Valdés M, Albornoz M, et al. Características clínicas e historia natural de la infección por virus de inmunodeficiencia humana. Rev Med Chile 1995; 123: 61-73.

2.- Stansell J D, Osmond D H, Charlebois E, Lavange L, Wallace JM, Alexander B V, et al. Predictors of Pneumocystis carinii pneumonia in HIV-infected persons. Pulmonary complications of HIV infection study group. Am J Respir Crit Care Med 1997; 155: 60-6.

3.- Hirschtick E, Glassroth J, Jordan M C, Wilcosky T, Wallace J, Kvale P, et al. Bacterial pneumonia in persons infected with the human immunodeficiency virus. N Engl J Med 1995; 333: 845-51.

4.- Wallace J M, Hansen N I, Lavange L, Glassroth J, Browdy B L, Rosen M J, et al. Respiratory disease trends in the pulmonary complications of HIV infection study cohort. Am J Respir Crit Care Med 1997; 155: 72-80.

5.- Fang G D, Fine M, Orloff J, Arisumi D, Yu V L, Kapoor W, et al. New emerging etiologies for community-acquired pneumonia with implications for therapy. A prospective multicenter study of 359 cases. Medicine (Baltimore) 1990; 69: 307-16.

6.- Murray P R, Washington J A II. Microscopic and bacteriologic analysis of expectorated sputum. Mayo Clin Proc 1975; 50: 339-44.

7.- Rabodonirina M, Raffenot D, Cotte L, Boibiex A, Mayencon M, Bayle G, et al. Rapid detection of Pneumocystis carinii in bronchoalveolar lavage specimens from human immunodeficiency virus-infected patients: Use of a simple DNA extraction procedure and nested PCR. J Clin Microbiol 1997; 35: 2748 51.

8.- Vuorinen P, Miettinen A, Vuento R, Hällström O. Direct detection of Mycobacterium tuberculosis complex in respiratory specimens by gen-probe amplified Mycobacterium tuberculosis direct test and Roche Amplicor Mycobacterium tuberculosis test. J Clin Microbiol 1995; 33: 1856-9.

9.- Whitby M, Kristinsson K G, Brown M. Assessment of rapid methods of pneumococcal antigen detection in routine sputum bacteriology. J Clin Pathol 1985; 38: 341-4.

10.- Palella F J Jr, Delaney K M, Moorman A C, Loveless M O, Fuhrer J, Satten G A, et al. Declining morbidity and mortality among patients with advanced human immunodeficiency virus infection. N Engl J Med 1998; 338: 796-800.

11.- Wolff A J, O`Donnell A E. Pulmonary manifestations of HIV infection in the era of highly active antiretroviral therapy. Chest 2001; 120: 1888-93.

12.- Feldman C. Pneumonia associated with HIV infection. Curr Opin Infect Dis 2005; 18 : 165-70.

13.- Wolff M J, Beltrán C J, Vásquez P, Ayala M X, Valenzuela M, Berríos G, et al. The Chilean AIDS cohort: a model for evaluating the impact of an expanded access program to antiretroviral therapy in a middle-income countryorganization and preliminary results. J Acquir Immune Defic Syndr 2005; 40: 551-7.

14.- Chin D P, Hopewell P C, Yajko D M, Vittinghoff E, Horsburg C R Jr, Hadley W K, et al. Mycobacterium avium complex in the respiratory or gastrointestinal tract and the risk of M. avium complex bacteremia in patients with human immunodeficiency virus infection. J Infect Dis 1994; 169: 289-95.

15.- Kalayjian R C, Toossi Z, Tomashefski J F Jr, Carey J T, Ross J A, Tomford J W, et al. Pulmonary disease due to infection by Mycobacterium avium complex in patients with AIDS. Clin Infect Dis 1995; 20: 1186-94.

16.- Mann M, Shelhamer J H, Masur H, Gill V J, Travis W, Solomon O, et al. Lack of clinical utility of bronchoalveolar lavage cultures for cytomegalovirus in HIV infection. Am J Respir Crit Care Med 1997; 155: 1723-8.

17.- Miles P R, Braughmann R P, Linnemann C C Jr Cytomegalovirus in the bronchoalveolar lavage fluid of patients with AIDS. Chest 1990; 97: 1072-6.

18.- Incidencia de algunas enfermedades de declaración obligatoria según sexo y región. 2005. www.minsal.cl (Temas de Salud. Estadísticas).

19.- Tarp B, Jensen J S, Østergaard L, Andersen P L. Search for agents causing atypical pneumonia in HIV-positive patients by inhibitor-controlled PCR assays. Eur Respir J 1998; 13: 175-9.

20.- Wolff A J, O'Donnell A E. HIV-related pulmonary infections: a review of the recent literature. Curr Opin Pulm Med 2003; 9 : 210-4.

21.- Chernilo S, Trujillo S, Kahn M, Paredes M, Echeverría G, Sepúlveda C. Enfermedades pulmonares en pacientes infectados con VIH hospitalizados en el Instituto Nacional del Tórax. Rev Med Chile 2005; 133: 517-24.

22.- Cruciani M, Marcati P, Malena M, Bosco O, Serpelloni G, Mengoli. Meta-analysis of diagnostic procedures for Pneumocystis carinii pneumonia in HIV-1-infected patients. Eur Respir J 2002; 20: 982-9.

23.- Leigh T R, Kangro H O, Gazzard B G, Jeffries D J, Collins JV. DNA amplification by the polymerase chain reaction to detect subclinical Pneumocystis carinii colonization in HIVpositive and HIV-negative male homosexuals with and without respiratory symptoms. Respir Med 1993; 87: 525-9.

24.- Wakefield A E, Lindley A R, Ambrose H E, Denis C M, Miller R F. Limited asymptomatic carriage of Pneumocystis jiroveci in human immunodeficiency virus-infected patients. J Infec Dis 2003; 187: 901-8.

25.- Morris A, Kingsley L A, Groner G, Lebedeva I P, Beards C B, Norris K A. Prevalence and clinical predictors of Pneumocystis colonization among HIV-infected men. AIDS 2004; 18: 793 8.

26.- Morris A, Wei K, Afshar K, Huang L. Epidemiology and clinical significance of Pneumocystis colonization. J Infect Dis 2008; 197: $10-7$

27.- Elvin K, Olsson M, Lindmann C, Bjorkmann A. Detection of asymptomatic Pneumocystis carinii infection by polymerase chain reaction: predictive for subsequent pneumonia. AIDS 1996; 10: 1296-7.

28.- Larsen H, Huang L, Kovacs J A, Crothers K, Silcott V A, Turner Jr, et al. A prospective, blinded study of quantitative touch-down polymerase chain reaction using oral-wash samples for diagnosis of Pneumocystis pneumonia in HIV-infected patients. J Infect Dis 2004; 189: 1679-83. 